\title{
The Relationship between the Size and the Invasion Depth of Tumors in Head and Neck Cutaneous Squamous Cell Carcinoma
}

\author{
Sam Yong Lee, Won Joo Hwang, Kyung Pil Kim, Hong Min Kim, Jae Ha Hwang, \\ Kwang Seog Kim \\ Department of Plastic and Reconstructive Surgery, Chonnam National University Medical School, Gwangju, Korea
}

Background Cutaneous squamous cell carcinoma (SCC), which occurs in keratinocytes of the epidermis and is the second most common skin cancer, has a more invasive growth pattern and higher potential to metastasize than basal cell carcinoma. Total excision of the primary tumor is the treatment of choice. For clear excision of the tumor, invasion depth is one of the most important factors. This study was conducted to clarify the relationship between the size and the invasion depth of cutaneous SCC.

Methods Twenty-six cases were collected for this prospective study. Frozen biopsies were examined after complete resection of the tumor, followed by histological confirmation by pathological examination. The major and minor axis lengths of the tumor, the invasion depth, and the level of invasion were measured. Recurrence or metastasis was recorded through regular follow-up.

Results The Pearson correlation coefficient was used for statistical analysis. Significant results were observed for the relationship between the major and minor axis lengths and the invasion depth of the tumor $(0.747,0.773)$. No cases of recurrence or metastasis were observed.

Conclusions In head and neck cutaneous SCC, the invasion depth of the tumor is closely related to the major and minor axis lengths of the tumor. Therefore, the invasion depth of the tumor can be estimated by measuring the size of the tumor, and a standard vertical safety margin for head and neck cutaneous SCC can be established, which could be helpful in the development of a preoperative reconstruction plan.

Keywords Carcinoma / Epithelial cells / Skin neoplasms / Keratinocytes
Correspondence: Sam Yong Lee Department of Plastic and Reconstructive Surgery, Chonnam National University Medical School, 160 Baekseo-ro, Dong-gu, Gwangju 61469, Korea

Tel: +82-62-220-6361

Fax: +82-62-227-1639

E-mail: sylee@jnu.ac.kr

This article was presented as a poster at the 73rd Congress of the Korean Society of Plastic and Reconstructive Surgeons, November 13-15, 2015 in Seoul, Korea.

No potential conflict of interest relevant to this article was reported.

\section{INTRODUCTION}

Cutaneous squamous cell carcinoma (SCC) is a typical nonmelanoma skin cancer occurring in keratinocytes and the second most common skin cancer. It has a more invasive growth pattern, higher recurrence rate, and higher potential to metastasize than basal cell carcinoma (BCC) [1]. It develops as a result of exposure to sunlight and ultraviolet radiation, scars caused by burns or injuries, chronic ulcer, extension of life expectancy, compromised immune status, and genetic defects [2]. 
According to the Korea National Statistical Office, the average life expectancy of South Koreans has increased from 78.6 years in 2005 to 81.94 years in 2015 and has continued to rise [3]. Accordingly, the prevalence rates for SCC have also increased.

In principle, the treatment of SCC is complete resection of the primary tumor [4]. Thus far, clinicians have focused on examination of the horizontal extensibility for determining the margin of complete resection; a safety margin has been emphasized as a therapeutic strategy for treatment without recurrence. Therefore, although research has been conducted on the tumor size and rate of metastasis to surrounding tissues in cases of head and neck squamous cell carcinoma (HNSCC), no study on metastasis according to the depth of tumor invasion has been reported.

The frequency of primary SCC has increased with population aging, and the recurrence rate has also increased in accordance with extending survival time after primary resection. Thus, there is a need for qualitative analysis of the depth of tumor resection by analyzing the resection site and the depth of invasion according to the tumor size.

This study hypothesized that the horizontal size of HNSCC was proportional to its vertical depth in the South Korean population. Establishing a relationship between the horizontal and vertical safety margins of SCC through the confirmation of this hypothesis could increase treatment completion.

\section{METHODS}

A prospective study was conducted on patients diagnosed with SCC who underwent surgical treatment in the Department of
Plastic and Reconstructive Surgery at our hospital between November 2014 and October 2015. This study examined age, gender, presence or absence of primary and recurrent tumors, presence or absence of complications, and reconstruction methods.

\section{Surgical methods}

All surgeries were performed under general anesthesia. In patients with primary tumors, the resection margin was designed, and complete resection was performed after setting the safety margin to approximately $7 \mathrm{~mm}$. The tumors were excised to a minimum 5-mm depth from the tumor base by including the dermis completely, and they were resected with inclusion of periosteum and cartilage. Specimens were obtained from the superior, inferior, medial, and lateral resection margin and base after resection for frozen section biopsy. When positive results were obtained, excision was performed to an additional 5-mm depth and width until negative results were obtained (Fig. 1A).

Regarding recurrent tumors, complete resection was performed by setting the safety margin to approximately $10 \mathrm{~mm}$. Additional resection was performed with the same method used for primary SCC. The residual defects after complete removal of the tumors were reconstructed using local flaps, including transposition flaps, island flaps, rotation flaps, and advancement flaps, by maintaining facial aesthetic units as much as possible (Fig. 1B). All patients were discharged an average of 2.3 days after surgery, and all surgical sutures were removed within 5 days after surgery.

Follow-up was conducted on an outpatient basis every 1, 2, 4, and 6 months and 1,2, and 3 years after discharge. Macroscopic examination and computed tomography were performed to ex-

\section{Fig. 1. Surgical procedure}

(A) Preoperative photo: designed excisional margin with safety margin. (B) Postoperative photo: covered defect with rotation flap.
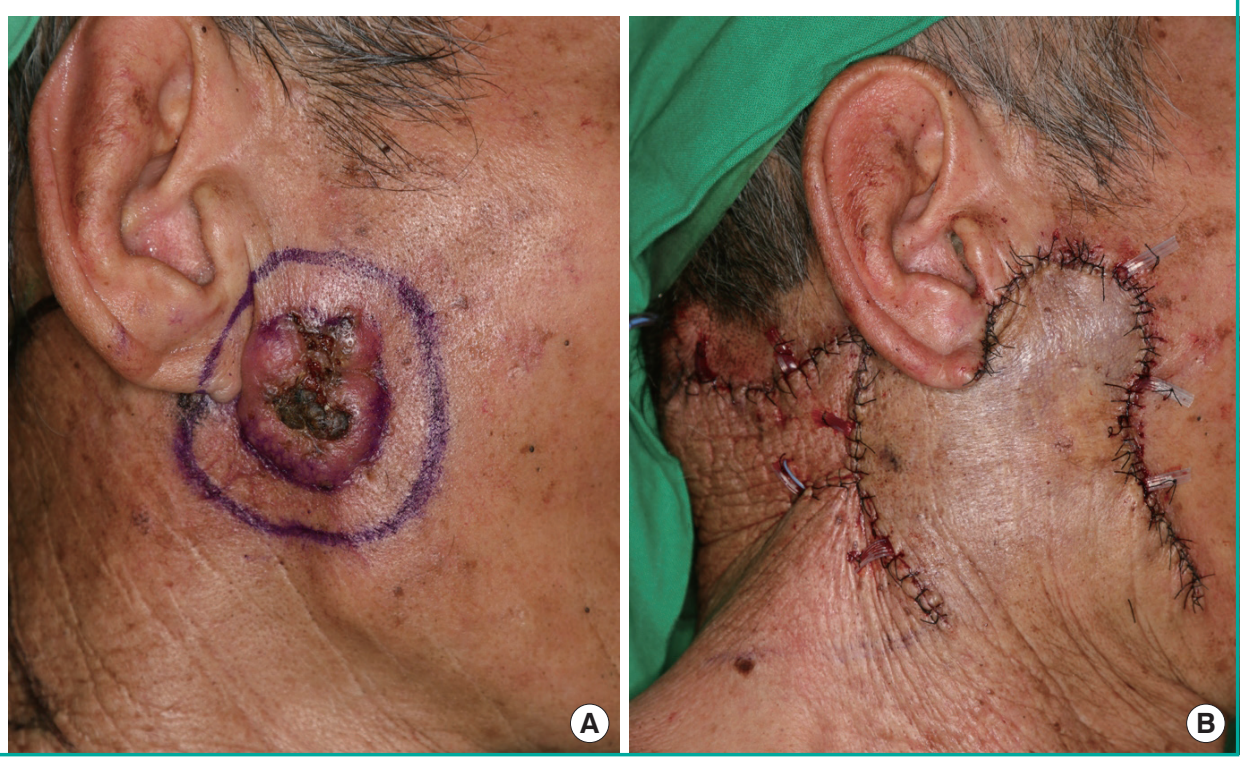
amine recurrence and sequelae. The mean follow-up period was 8.9 months.

\section{Pathological methods}

The major and minor axis lengths of the surgically resected specimens were measured using a microscopic ruler, and then fixed by formalin for 24 hours. Paraffin blocks were prepared by serial sectioning of the fixed tissue at 2-mm intervals, followed by the preparation of slides. Each slide was dewaxed using $x y-$ lene; hematoxylin and eosin staining was then applied. The stained slide was evaluated by the horizontal size of SCC (major axis $\times$ minor axis, $\mathrm{mm}$ ), the depth of tumor ( $\mathrm{mm}$ ), and invasion into the surrounding tissues based on microscopic pathological findings (Fig. 2).

The major axis of the tumor, as identified by this study, represents the longest horizontal length of the tumor, while the minor axis represents the longest horizontal length perpendicular to the major axis. The size is defined as the product of the values of the major axis and minor axis; the invasion depth is defined as the deepest vertical length of the tumor measured by a microscope; and the invasion layer is defined as the deepest tissue layer invaded by the tumor (e.g., dermis, subcutaneous tissue, and muscle).

\section{Statistical methods}

Statistical analysis was performed with the SPSS software package, ver. 21.0 (IBM Corp., Armonk, NY, USA). The statistical correlation between the major and minor axis lengths of the tumor and the depth of tumor invasion ( $\mathrm{mm}$ ) was evaluated using the Pearson correlation coefficient. The invasion was classified according to dermis, subcutaneous layer, and muscle layer, and then evaluated by the correlation between the major and minor axis lengths of the tumor. A P-value of $<0.05$ was considered a statistically significant result.

\section{Fig. 2. Tumor measurements}

$a$, horizontal safety margin; $b$ major axis of tumor; $c$, minor axis of tumor; $d$, invasion depth. Large circle $(0)$ and smaller circle $(\bullet)$ indicate excision margin and tumor margin, respectively.

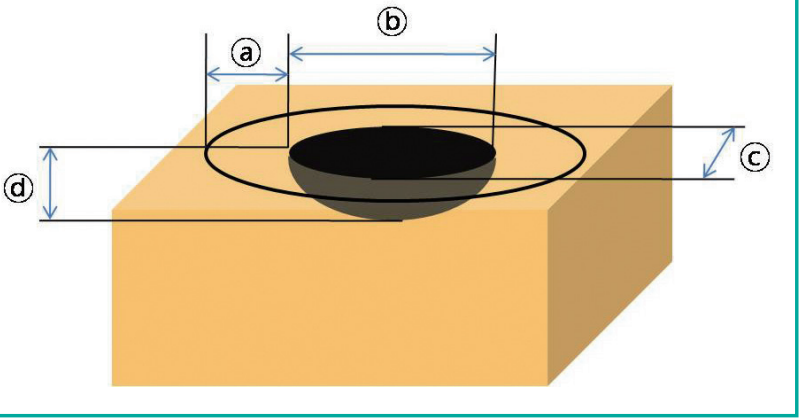

\section{RESULTS}

Data were collected from 26 patients, with a mean age of 81.2 years, including 11 males and 15 females. The mean tumor size was $2.49 \times 2.03 \mathrm{~cm}^{2}$. Eighteen patients had primary tumors and 8 patients had recurrent tumors after undergoing treatment such as laser therapy or simple excision. All carcinomas were completely removed after surgery, and there were no complications such as hematoma, seroma, partial, or total flap necrosis after reconstructive surgery. There were no patients with recurrent tumors during follow-up (Table 1).

\section{Correlation between major axis length of tumor and depth of tumor invasion}

The relationship between the major axis length of the tumor and the depth of tumor invasion was proportional, with a Pearson correlation coefficient of $0.747(\mathrm{P}<0.001)$, and showed statistically significant results (Table 2). No difference was observed between primary and recurrent carcinomas.

\section{Correlation between minor axis length of tumor and depth of tumor invasion}

In a comparison of the relationship between the minor axis

\section{Table 1. Patient characteristics}

\begin{tabular}{|lc|}
\hline Characteristic & $\begin{array}{c}\text { No. of patients } \\
(\mathbf{n}=\mathbf{2 6})\end{array}$ \\
\hline Mean age (yr) & $81.2(65-94)$ \\
Male:Female ratio & $11: 15$ \\
Mean major axis of tumor gross length $(\mathrm{cm})$ & $2.5(0.6-5.7)$ \\
Mean minor axis of tumor gross length (cm) & $2.0(0.3-5.4)$ \\
Mean invasion microscopic depth (mm) & $7.2(2-25)$ \\
Invasion layer ratio (dermis:subcutaneous tissue:muscle) & $18: 5: 3$ \\
Primary tumor:Recurred tumor ratio & $18: 8$ \\
Complication (hematoma, seroma, necrosis, etc.) & 0 \\
Recurrence & 0 \\
\hline The values given in parentheses are the minimum and maximum values of the \\
corresponding item.
\end{tabular}

Table 2. Relation between tumor size an invasion depth and layer

\begin{tabular}{|lcc|}
\hline Variable & Invasion depth & Invasion layer \\
\hline Major axis of tumor & & \\
$r$ & 0.747 & 0.032 \\
P-value & $<0.001$ & 0.881 \\
No. & 26 & 26 \\
Minor axis of tumor & & \\
$r$ & 0.773 & -0.050 \\
P-value & $<0.001$ & 0.815 \\
No. & 24 & 24 \\
\hline
\end{tabular}


length of tumor and depth of tumor invasion, the Pearson correlation coefficient of $0.773(\mathrm{P}<0.001)$ was proportional, and showed statistically significant results (Table 2 ). No difference was observed between primary and recurrent carcinomas.

\section{Correlation between horizontal tumor size and layer of tumor invasion}

According to the classification of invasion, more than half of specimens (18 patients) were localized in the dermis (Table 1). The relationship between the major and minor axis lengths of the tumor and invasion showed no statistically significant results (Table 2).

Linear regression analysis was performed using the proportion between the major and minor axis lengths of the tumor and the depth of invasion (Figs. 3, 4).

$\mathrm{Y}(\mathrm{mm}$, depth of invasion $)=2.805 \times \mathrm{X}(\mathrm{cm}$, length of major axis) $+0.118, \mathrm{r}^{2}=0.558(1)$

$\mathrm{Y}(\mathrm{mm}$, depth of invasion $)=3.131 \times \mathrm{X}(\mathrm{cm}$, length of minor axis) $+0.704, r^{2}=0.597(2)$

In a comparison of the difference between the values of $Y$ (the measured depth of invasion) obtained by substituting the major and minor axis lengths into value $\mathrm{X}$ of the 2 completed equations, Eq. (2) with $X=$ length of minor axis showed higher similarity.

\section{DISCUSSION}

Cutaneous SCC, a typical non-melanoma skin cancer with $\mathrm{BCC}$, shows aggressive features, with a high rate of metastasis at

\section{Fig. 3. Scatter plots (major axis)}

Scatter plots with regression line between tumor major axis length and tumor invasion depth.

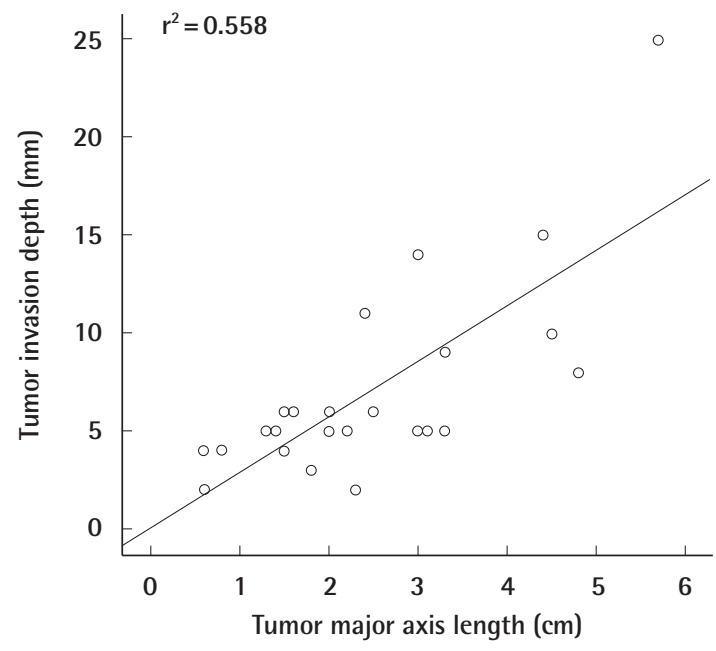

$12.5 \%$ [5]. It develops due to exposure to sunlight and ultraviolet radiation, scars caused by burns or injuries, chronic ulcer, extension of life expectancy, compromised immune status, and genetic defects [2]. Although the reported frequency is 2.6 to 2.9 per 100,000 population [6], it will gradually increase with extensions to life expectancy.

Surgical resection is the treatment of choice for SCC, and nonsurgical treatments include cryotherapy, curettage and electrodesiccation, and radiation therapy. Nonsurgical treatments, however, are applied to patients who have difficulty in undergoing surgery due to a high failure rate in recurrent tumors or large tumors; these nonsurgical treatments have a high recurrence rate $[7,8]$.

Surgical resection in SCC is based on tumor size. Surgical resection is performed to include the tumor and a surrounding safety margin. However, controversy remains over what constitutes an adequate safety margin. Brodland and Zitelli [4] recommend $4 \mathrm{~mm}$ for a low-risk tumor and $6 \mathrm{~mm}$ for a high-risk tumor. Guidelines from the National Comprehensive Cancer Network recommend 4-6 $\mathrm{mm}$ for low-risk tumors and $10 \mathrm{~mm}$ for high-risk tumors [9]. Based on the surgeon's experience, our hospital has set $7 \mathrm{~mm}$ as the safety margin for primary tumor cases and $10 \mathrm{~mm}$ as the safety margin for secondary tumor cases. However, what is most important is that the excision have clean margins. Invasion of tumor cells on the resection margin is confirmed by pathological examination. When positive results are obtained, additional resection should be performed to check for negative results $[4,9]$. The low- and high-risk groups are classified according to tumor size and location, histological classifi-

\section{Fig. 4. Scatter plots (minor axis)}

Scatter plots with regression line between tumor minor axis length and tumor invasion depth.

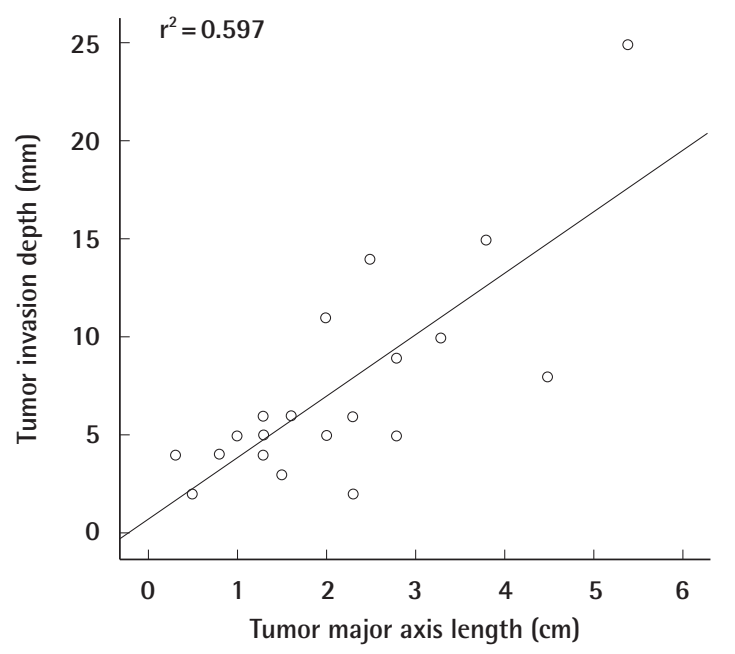


Table 3. Factors associated with high risk for recurrence in squamous cell carcinoma

\begin{tabular}{|ll|}
\hline High risk tumor characteristics & \multicolumn{1}{c|}{ High risk size/location combinations } \\
\hline Poorly defined borders & $\geq 6 \mathrm{~mm}$ in "mask areas" of the face (central face, eyelids, eyebrows, nose, \\
& $\begin{array}{l}\text { periorbital, lips, chin, ear, pre/post auricular skin, mandible, temple), genitalia, hands/ } \\
\text { feet }\end{array}$ \\
$\begin{array}{l}\text { Recurrent lesion } \\
\text { Immunosuppressed patient }\end{array}$ & $\geq 10 \mathrm{~mm}$ in the scalp, forehead, cheeks, neck \\
$\begin{array}{l}\text { Site of prior radiotherapy or chronic inflammation (marjolin's ulcer) } \\
\text { Rapidly growing tumor }\end{array}$ & \\
$\begin{array}{l}\text { Neurologic symptoms } \\
\text { Certain pathologic characteristics (perineural or vascular involvement, poor } \\
\text { differentiation, adenoid, adenosquamous or desmoplastic subtypes, thickness } \geq 2 \\
\text { mm or clark level IV or V) }\end{array}$ & \\
\hline
\end{tabular}

cation, tumor characteristics, and invasion of surrounding tissues (Table 3) [9].

When the depth of SCC reaches the subcutaneous tissues or beyond, the metastasis rate increases dramatically $[10,11]$. Some doctors believe that tumor depth is the most important factor in predicting metastasis of SCC [11]. Moreover, since the tumor relapse rate is higher at greater depth, it is important to identify the depth of a tumor during treatment.

Five-year recurrence of cutaneous SCC varies depending on tumor size. It reaches approximately $8 \%$ and $15 \%$ with small (diameter $<2 \mathrm{~cm}$ ) and large (diameter $>2 \mathrm{~cm}$ ) tumors, respectively [12-14]. Regarding recurrent tumors, the recurrence rate is double, and the rate of local metastasis reaches $5 \%$ to $30 \%$ [12].

With the increase in life expectancy, it is expected that not only incidence of SCC but also the recurrence rate will increase as the follow-up period after treatment is extended. Thus, complete treatment of primary SCC is important.

Incomplete resection has been evaluated as the most common cause of an increase in tumor recurrence and metastasis risk in the treatment of SCC $[12,15]$. In other words, there is a need for complete resection considering tumor extension in order to lower the recurrence rate. To this end, a safety margin is important. However, current standards of care for SCC specify only the horizontal safety margins. Standards considering the depth of tumor invasion have yet to be established, and no research has been conducted on the relationship between horizontal and vertical extension of tumors and metastasis according to vertical extension of tumors. Therefore, it is difficult to clarify whether recurrence due to incomplete resection is caused by the remainder of the tumor on the horizontal resection margin or the remainder of tissue on the tumor base.

In this study, serial section slides were prepared after surgical resection according to existing guidelines for patients with SCC, and data were collected by pathological examination of the base depth in each slide. With these data, the depth of the tumor base was quantified by mapping based on the total horizontal area of the tumor. Statistical analysis of the correlation between the major and minor axis lengths of the tumor and the maximum depth of the tumor base was performed. A proportional relationship between the major axis length of the tumor and the depth of tumor invasion was demonstrated with a Pearson correlation coefficient of 0.747 .

A proportional relationship of the minor axis length of the tumor and the depth of tumor invasion was also demonstrated with a Pearson correlation coefficient of 0.773 . It can be concluded that horizontal growth and vertical growth of the cutaneous SCC have a proportional relationship. This accords with our previously described hypothesis.

The following linear regression equations were obtained by performing a regression analysis based on the horizontal tumor size (major and minor axis lengths) measured using a microscope:

$\mathrm{Y}(\mathrm{mm}$, depth of invasion $)=2.805 \times \mathrm{X}(\mathrm{cm}$, length of major axis) $+0.118, \mathrm{r}^{2}=0.558(1)$

$\mathrm{Y}(\mathrm{mm}$, depth of invasion $)=3.131 \times \mathrm{X}(\mathrm{cm}$, length of minor axis) $+0.704, \mathrm{r}^{2}=0.597(2)$

For example, a 7.13-mm depth of invasion is obtained when substituting $2.5 \mathrm{~cm}$ as the average measured length of the major axis of the patient into Eq. (1). A 6.97-mm depth of invasion is obtained when substituting $2.0 \mathrm{~cm}$ as the average measured length of the minor axis of the patient into Eq. (2), and this value is similar to $7.2 \mathrm{~mm}$, the measured average depth of invasion. Therefore, the use of this equation makes it possible to estimate the depth of the tumor base by measuring the horizontal tumor size. This means that a depth of at least $7.13 \mathrm{~mm}$ should be applied for complete resection for patients with SCC measuring $2.5 \times 2.0 \mathrm{~cm}$. This also makes it possible to set a vertical safety margin in proportion to tumor size.

A limitation of the present study is that the data were drawn from 26 patients, which may not be sufficient for generalization 
with the formulae obtained. However, the correlations between the data were statistically significant and the derived formulae are expected to be validated when applied in future to other patients.

In order to perform complete resection of SCC, many plastic and reconstructive surgeons and doctors have focused on the horizontal safety margin and surrounding tissues. In this study, we clarified the proportional relationship between the horizontal size and vertical depth of SCC and provided formulae for determining the vertical safety margin. Performance of resection in consideration of the horizontal and vertical safety margins of tumors based on these results could not only reduce risks such as tumor recurrence by complete resection of the tumor base, but also minimize the defect size of surgical sites by removing appropriate tissues.

\section{REFERENCES}

1. Johnson TM, Rowe DE, Nelson BR, et al. Squamous cell carcinoma of the skin (excluding lip and oral mucosa). J Am Acad Dermatol 1992;26:467-84.

2. Chung HG, Moon TK, Bang DS, et al. Clinical observation of cutaneous malignant tumors and premalignant lesions over 15 years (1982-1996). Korean J Dermatol 1999;37: 1413-22.

3. Statistics Korea. A mortality table, national approval statistics, no. 10135. Daejeon: Statistics Korea; 2013.

4. Brodland DG, Zitelli JA. Surgical margins for excision of primary cutaneous squamous cell carcinoma. J Am Acad Dermatol 1992;27:241-8.

5. Zheng Z, Kye Y, Zhang X, et al. Expression of p63,bcl2,bcl-6 and p16 in basal cell carcinoma and squamous cell carcinoma of the skin. Korean J Pathol 2005;39:91-8.

6. Takemiya M, Ohtsuka H, Miki Y. The relationship between solar keratoses and squamous cell carcinomas among Japa- nese. J Dermatol 1990;17:342-6.

7. Motley R, Kersey P, Lawrence C. Multiprofessional guidelines for the management of the patient with primary cutaneous squamous cell carcinoma. Br J Dermatol 2002;146: $18-25$.

8. Freeman RG, Knox JM, Heaton CL. The treatment of skin cancer: a statistical study of 1,341 skin tumors comparing results obtained with irradiation, surgery, and curettage followed by electrodesiccation. Cancer 1964;17:535-8.

9. Bichakjian CK, Olencki T, Aasi SZ, et al. NCCN clinical practice guidelines in oncology (NCCN Guidelines). Squamous cell skin cancer, version 1.2015 [Internet]. Fort Washington: National Comprehensive Cancer Network, Inc.; 2015 [cited 2015 Mar 25]. Available from: http://nccn.org.

10. Kwa RE, Campana K, Moy RL. Biology of cutaneous squamous cell carcinoma. J Am Acad Dermatol 1992;26:1-26.

11. Bernstein SC, Lim KK, Brodland DG, et al. The many faces of squamous cell carcinoma. Dermatol Surg 1996;22:24354.

12. Rowe DE, Carroll RJ, Day CL Jr. Prognostic factors for local recurrence, metastasis, and survival rates in squamous cell carcinoma of the skin, ear, and lip. Implications for treatment modality selection. J Am Acad Dermatol 1992;26: 976-90.

13. Czarnecki D, Staples M, Mar A, et al. Metastases from squamous cell carcinoma of the skin in southern Australia. Dermatology 1994;189:52-4.

14. Jackson A. Prevention, early detection and team management of skin cancer in primary care: contribution to The health of the nation objectives. Br J Gen Pract 1995;45:97101.

15. Cherpelis BS, Marcusen C, Lang PG. Prognostic factors for metastasis in squamous cell carcinoma of the skin. Dermatol Surg 2002;28:268-73. 